\title{
Predicción de la influencia del voto duro sobre el voto nulo en un sistema electoral democrático de dos partidos
}

\author{
Prediction of the influence of core vote on null \\ vote in a democratic two-party electoral system

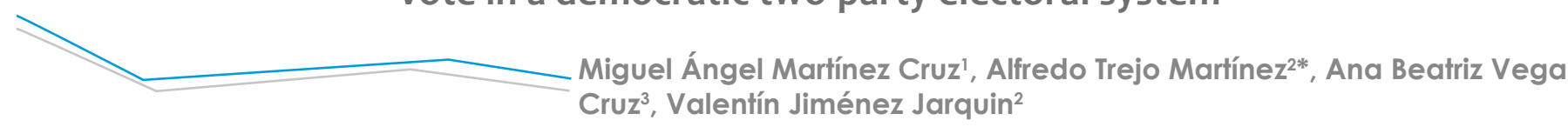 \\ Martínez Cruz, M. A., Trejo Martínez, A., Vega Cruz, A. B., Jiménez Jarquin, V. Predicción de la \\ influencia del voto duro sobre el voto nulo en un sistema electoral democrático de dos partidos. \\ Investigación y Ciencia de la Universidad Autónoma de Aguascalientes. Número 68: 78-85 \\ mayo-agosto 2016.
}

En este trabajo se analiza el efecto que podría ejercer el voto duro sobre el voto nulo en un sistema electoral de dos partidos, mediante la introducción de una red de opinión con agentes activos (nodos) distribuidos uniformemente en su estado inicial con un valor denominado spin $s=+1, s=-1$ ó $s=0$. Los dos primeros valores, llamados votos duros, representan a los simpatizantes incondicionales de una corriente política elegida; el último representa a los votantes inactivos o indecisos, los cuales podrían cambiar a $s=+1$ ó $s=-1$ por las interacciones con sus cuatro conocidos más cercanos, denominados 4-vecinos. Se realizaron tres estudios: primero se analizó la relación de la concentración final de agentes activos con respecto a su cantidad inicial, posteriormente se observó la proporción final de agentes inactivos con respecto a la proporción inicial de agentes activos, finalmente se observa la evolución del sistema en relación con el número de ciclos que transcurren para alcanzar la estabilidad.

Palabras clave: red de opinión, simulación, red de spin, voto duro, voto nulo, red electoral.

Keywords: opinion network, simulation, spin network, core vote, null vote, electoral network.

\section{Recibido: 18 de febrero de 2015, aceptado: 8 de febrero de 2016}

1 Departamento de Sistemas, Sección de Estudios de Posgrado e Investigación, ESIME-Zacatenco-IPN.

2 Área de Estudios de Posgrado e Investigación, Tecnológico de Estudios Superiores del Oriente del Estado de México.

3 Universidad Autónoma del Estado de México Unidad Académica Profesional Chimalhuacán.

* Autor para correspondencia: aalfredoł@yahoo.com.mx
In this work we analyze the possible effect that the core vote might have on the null vote in a two-party system, by introducing an opinion network with active agents (nodes) uniformly distributed in their initial state with a value called spin $s=+1$, spin $s=-1$, and spin $s=0$. The first two values, called core votes, represent staunch supporters of a chosen political current; the last value represents inactive or undecided voters which might change to $s=+1$ or $s=-1$ due to the interactions with their four closest acquaintances, also known as 4-neighbors. We conducted three studies. First we analyzed the relationship between the final concentration of active agents and the initial proportion of active agents, then we observed the final proportion of inactive agents with respect to the initial proportion of active agents, and finally, we observed the system evolution, with respect to the number of cycles that elapse before the system reaches stability.

INTRODUCCIÓN

El voto es una manifestación de la propia voluntad u opinión de una persona o grupo de personas acerca de un tema y regulada por alguna entidad que se emite en forma normada, sin fundamentación explícita y encaminada a una decisión colectiva. Una votación implica la existencia de un grupo de personas habilitadas para votar y de un asunto previamente delimitado sobre el cual se expresará la voluntad de los integrantes del grupo. Adjei (2012) define al voto como una expresión oficial de la elección de los votantes de una de las posibilidades ofrecidas o propuestas, regularmente marcando una boleta, de manera secreta. Puede ser considerado como un proceso de decisión en el cual los votantes son los decisores y 
los partidos o candidatos son las alternativas. El voto puede ser secreto o público, directo o indirecto, simple (un votante, un voto), plural (un votante, varios votos) o acumulativo (tantos votos como candidatos a elegir). Cuando se vota por una de las opciones que se da a elegir lo llamamos voto activo.

La existencia de partidos políticos es el mejor medio para ejercer la democracia (Rosenblum, 2008). Los partidos resuelven problemas de toma de decisiones colectivas en las cámaras en el proceso de creación de las leyes (Aldrich, 1995). Ayudan a decidir a los votantes que no tienen tiempo o la determinación para informarse acerca de todos los candidatos y sus propuestas (Popkin, 1991). Forman una estructura electoral con una serie de opciones estables que disminuyen la incertidumbre en el proceso electoral, hacen que este se vuelva más predecible y extiende el horizonte de tiempo de los políticos (Mainwaring y Scully, 1995). Inculcan disciplina en los políticos y los instan a rendir cuentas (Alesina y Spear, 1988).

Rae (1969) define a los sistemas electorales como un conjunto de leyes que rigen los procesos por los cuales las preferencias se traducen en votos y estos votos se traducen en la elección de los gobernantes - partidos; afirma que los sistemas electorales rigen los procesos electorales en su totalidad, incluyendo al propio sufragio, los requisitos para el registro de partidos, la distribución de los distritos y el financiamiento de las campañas.

El modelo propuesto en este trabajo es aplicable a sistemas electorales de dos partidos. Lijphart (1994) menciona algunas ventajas de los sistemas de dos partidos; argumenta que tienen mayor estabilidad y centralismo político, lo cual puede provocar un crecimiento económico, son más fáciles de gobernar, tienen una menor propensión a la indecisión en las cámaras y menor divisibilidad política, el proceso de decisión de los votantes es menos complejo debido a la existencia de dos alternativas, se genera una menor interferencia en las políticas económicas; sin embargo, afirma que sus desventajas son que existe una menor competitividad partidaria, podrían generar apatía en los votantes ya que puede provocar una percepción de falta de alternativas, condicionan a los votantes a sólo dos opciones, impidiendo la libertad de elección, limitan la existencia de diversos puntos de vista en el gobierno.

Según Sirvent y Delgadillo (1997) diversos estudios empíricos sobre el comportamiento del electo- rado en las democracias modernas han demostrado que existen segmentos de la población con considerable estabilidad en sus preferencias, lo cual indica un voto individual con alta predictibilidad en su comportamiento político a lo largo del tiempo. Giddens (1996) define este fenómeno como el comportamiento electoral, agrega que se puede describir como la lealtad que tienen los votantes hacia uno u otro candidato o partido en el proceso electoral. De ahí que se hable de identificación partidista estable, también conocida como voto duro, lo cual significa que los partidos políticos cuentan con una clientela electoral estable derivada de las condiciones de su estructura. En el otro extremo encontramos el abstencionismo y voto nulo, ambos manifiestan el desacuerdo o indiferencia de los votantes hacia las ideas de los candidatos propuestos. Por un lado, en la abstención, el votante decide no ejercer su derecho al voto, a diferencia del voto nulo, en donde el votante decide anular de forma cuantitativa cualquier preferencia por los candidatos competidores.

En los sistemas políticos democráticos se realizan campañas para la anulación del voto, una de sus razones principales es el reclamo de los votantes debido a su inconformidad con las ideas de los participantes propuestos. Finalmente el elector realiza una crítica política meditada y muchos optan por la anulación del voto de manera intencional. Existe la anulación del voto de manera no intencional, lo cual no es considerado en esta investigación.

Muchas de las características de los sistemas sociales son independientes de las opiniones particulares de cada individuo y de los detalles de las interacciones sociales que tengan. Ello permite que al modelar el comportamiento global se puedan utilizar las herramientas de la física estadística. Las hipótesis de los modelos de opinión se basan en la observación social que la gente tiende a realizar al intercambiar sus puntos de vista, y son estas interacciones la causa del cambio de opinión hacia un consenso entre toda la sociedad (Wasserman y Faust, 1994; Wilheim, 2000). De esta manera, los modelos estocásticos de redes de spin son ampliamente utilizados para estudiar las características generales de formación de la opinión social (Travieso y Costa, 2006; Pabjan y Pekalski, 2008; Toivonen et al., 2009). Knoke y Yang (2008) definen el concepto de red como un grupo de objetos, también denominados nodos o vértices, relacionados 0 unidos entre ellos por medio de arcos. En una red social, los nodos representan a las personas y los arcos representan las relaciones que se presentan 
entre las personas; esas relaciones pueden ser de amistad, parentesco, colaboración, información, entre otras. La decisión del voto puede cambiar o permanecer constante como consecuencia de dichas relaciones.

En general, los modelos de spin se componen de agentes activos que se fijan en los vértices de la red, cada agente puede asumir algún estado y su evolución se rige por una dinámica en particular. En el caso concreto de nuestro trabajo cada spin puede tener alguno de los siguientes estados $s=+10$ $s=-1$, lo que corresponde a votantes con opiniones opuestas, o $s=0$, que determina un estado neutral o un votante indeciso, además, el sistema evoluciona de acuerdo a una dinámica específica, la cual provoca que tienda a una estabilización entre los grupos, en donde ningún votante más puede cambiar de opinión. Uno de los modelos más populares es el modelo de la regla mayoritaria (Regenwetter, 2002). Diferentes propiedades de la regla mayoritaria son tratadas por Tideman (1986), Regenwetter (2002), Miroiu (2004), Chen y Redner (2005), entre otros.

En el modelo de dinámica de opinión estable con tres estados, desarrollado en este trabajo, al interactuar un spin con sus vecinos, sólo el spin seleccionado cambia de estado; mientras que en el modelo de la regla mayoritaria el spin seleccionado junto con sus vecinos cambian al mismo estado.

El modelo de la regla mayoritaria fue utilizado por primera vez en un simple modelo estadístico geométrico que muestra una transición de fase continua (Tsallis, 1982). En el contexto de la formación de la opinión, la regla de la mayoría fue utilizada originalmente en un modelo sencillo que describe el comportamiento del voto dentro de una sociedad jerárquica (Galam, 1999). Además, se constató que la dinámica de los modelos de la regla mayoritaria es dependiente de la topología de la red. Se considera que la mayoría de los modelos de comportamiento del voto son modelos no equilibrados, en dos dimensiones. Y el mallado regular aplicado a los sistemas pertenece a la misma universalidad que la del modelo de equilibrio de Ising, debido a la simetría arriba-abajo (Vilela y Moreira, 2009).

Recientemente, se demostró que la existencia de sociedades bien estructuradas dentro de una red social puede afectar drásticamente la dinámica de formación de la opinión (Lambiotte et al., 2007; Huang et al., 2008). En este trabajo las sociedades son caracterizadas por diferentes condiciones sobre la conectividad de los agentes que estén dentro o fuera de esta. También estudiamos los efectos de la concentración inicial de los agentes activos e inactivos en la dinámica de la formación de opinión.

\section{MATERIALES Y METODOS}

Para modelar la dinámica de opinión utilizamos una red cuadrada homogénea con $N$ nodos conectados únicamente con sus cuatro vecinos de radio 1 . Cada nodo $n_{i} \in[1, N]$ puede tomar alguno de los tres estados $i=+1,-1$, 0 . En el contexto de la dinámica social, un nodo $n_{i}$ con spin $i \neq 0$ está asociado con agentes activos; es decir, votantes con alguna preferencia política, mientras que los nodos con spin $i=0$ están asociados con agentes inactivos o votantes no comprometidos o indecisos. Inicialmente los nodos con estado +1 y -1 son distribuidos con la condición de no cambiantes; es decir, voto duro.

Dentro del proceso de simulación en el cual la distribución inicial de los agentes activos es fijada de manera aleatoria, se guarda una relación definida por la Ecuación 1.

$$
\left(n_{0}\right)_{\% s}=N-\left(n_{+1}\right)_{\% s^{-}}\left(n_{-1}\right)_{\% s}
$$

Donde:

$\left(n_{0}\right)_{\% s}$ representa el porcentaje inicial de nodos con spin 0 (votantes indecisos).

$\left(n_{+1}\right)_{\% s}$ representa el porcentaje inicial de nodos con spin +1 (votantes con preferencia por el candidato A).

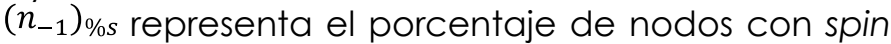
-1 (votantes con preferencia por el candidato B).

Una fase subsecuente de actualización (ciclo) con respecto a la distribución inicial se obtiene cuando son seleccionados $N$ nodos de la red de manera aleatoria y con reemplazo; cada nodo sólo interaccionará con sus cuatro vecinos más cercanos y podrá modificar su estado de preferencia inicial de acuerdo a la preferencia mayoritaria vecinal de la fase. Por otro lado, el estado de equilibrio es alcanzado cuando ningún votante al interactuar con sus vecinos cambia de opinión, lo que permite interpretar matemáticamente el efecto del voto duro sobre el voto nulo. En la Figura 1 se da muestra de la distribución inicial en una red de 50 × 50 con el $10 \%$ de spines +1 , en color negro, $10 \%$ de spines -1 , en color blanco y $80 \%$ de spines neutros, en color gris. 


\section{Reglas del proceso de simulación}

En el estado inicial los agentes son distribuidos uniformemente, de acuerdo a la corriente política emulada, todos dentro de una red cuadrada de tamaño variante, en este estado la suma de todos los agentes siempre es menor o igual al $100 \%$ del tamaño de la red. En una fase subsecuente de actualización cada agente es seleccionado aleatoriamente y con reemplazo, para analizar su posible cambio de estado de acuerdo al estado predominante entre sus cuatro vecinos. Este análisis individual define una iteración y $N$ iteraciones definen un ciclo $C$, cuando es alcanzado el primer ciclo el proceso se inicia nuevamente con condiciones distintas a las iniciales incrementando el número de ciclos $n$, este proceso evoluciona hasta alcanzar el estado de equilibrio; es decir, donde no haya más iteraciones posibles por realizar.

Es importante señalar que la regla empleada impide la formación de franjas, lo que en consecuen-

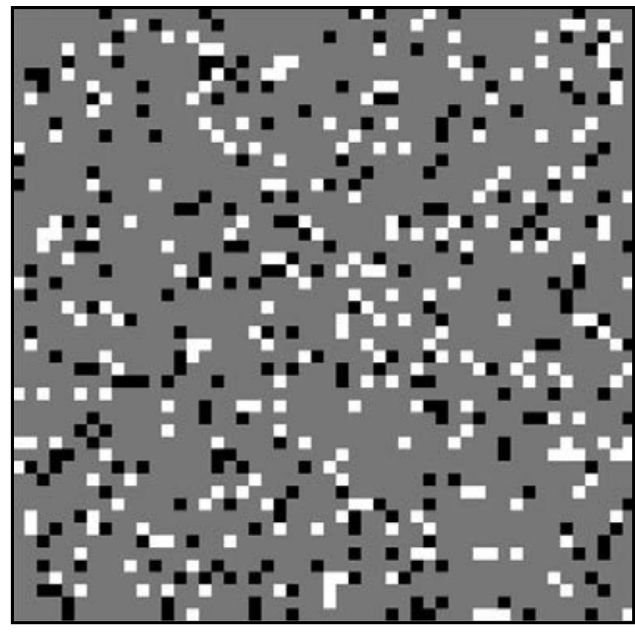

Figura 1. Estado inicial de una red de $50 \times 50$ con el $10 \%$ de spines +1 (negros), $10 \%$ de spines -1 (blancos) y $80 \%$ de nodos inactivos (grises). Imagen elaborada por los autores. cia evita que se tenga una cinética lenta y exista un consenso (Balankin et al., 2011). Esto marca la diferencia entre nuestra regla y la regla de la mayoría relativa propuesta por Galam (1999). En la Figura 2 se muestra la diferencia entre la actualización al aplicar la regla propuesta y la regla mayoritaria sobre una red cuadrada; a) muestra la actualización en una interacción de un nodo considerando su opinión y sus cuatro vecinos más cercanos aplicando la regla propuesta y b) muestra la actualización en una interacción de un nodo aplicando la regla mayoritaria.

Los resultados presentados en este trabajo se obtuvieron después de analizar un espacio muestral de tamaño 100, para distintas dimensiones de la red:

$N=400,900,1600,2500,3600,4900,6400,81000,10000,12100,14400$ y 1000000.

Considerando una muestra al dejar que el sistema evolucione y llegue a su estado de equilibrio; es decir, no haya más cambios de estado entre los agentes.

La Figura 3 presenta el estado final de una red de agentes, que su estado inicial estaba definido por el $10 \%$ de su tamaño con agentes en un estado de spines +1 (negro), otro $10 \%$ del tamaño de la red con agentes en estado de spines -1 (blanco) y el $80 \%$ de la población restante con agentes con estado spines neutros (gris).

RESULTADOS

\section{Comportamiento de agentes activos}

En primer lugar, nos concentramos en la relación de agentes iniciales y finales con spin +1 , realizando diversas pruebas establecidas mediante el porcentaje de agentes $n_{+1},\left(n_{+1}\right)_{\% \text { s, en un intervalo }}$ de $[0,0.5] N$ y manteniendo fijo el número de agentes $n_{-1}\left(n_{-1}\right) \% s$ en $0.5 \mathrm{~N}$, para distintos valores de $N$. El a)

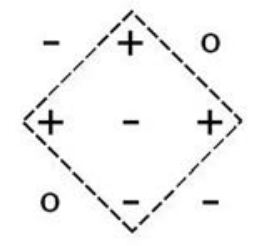

b)

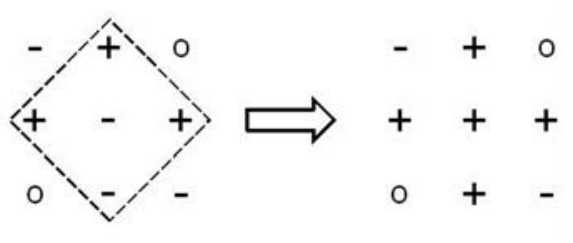

Figura 2. llustración de la diferencia entre la actualización de la aplicación de la regla mayoritaria y de la propuesta sobre una red cuadrada. 


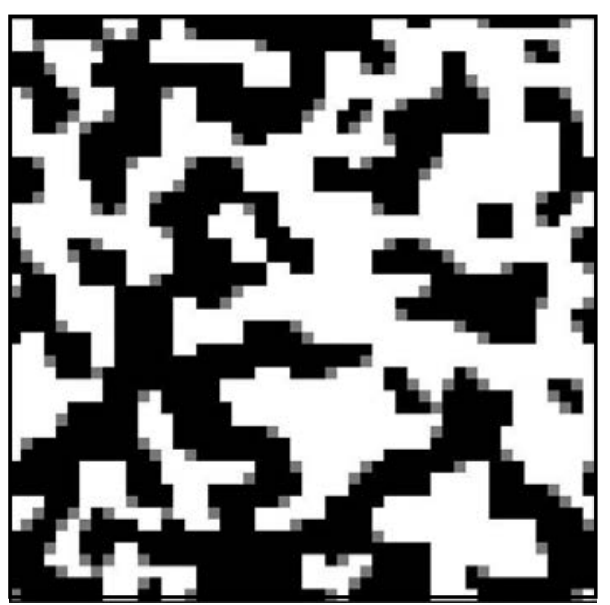

Figura 3. Estado final de una red de $50 \times 50$ con estado inicial de $10 \%$ de spines +1 (negros), $10 \%$ de spines -1 (blancos) y $80 \%$ nodos inactivos (grises).

porcentaje final de spin con estado +1 queda descrito por la Ecuación 2.

$$
\left(n_{+1}\right)_{\% f}\left[\left(n_{+1}\right)_{\% s}\right]=1.965\left(\left(n_{+1}\right)_{\% s}\right)^{1.8377}
$$

\section{Donde:}

$\left(n_{+1}\right) \% f$ representa el porcentaje de agentes con estado +1 obtenidos al llegar al estado de equilibrio. $\left[\left(n_{+1}\right)_{\% s}\right]$ representa el porcentaje de agentes con estado +1 distribuidos inicialmente.

La Figura 4, que se encuentra con escala logarítmica, describe un comportamiento estable de distribución de colas pesadas con respecto a $\left(n_{+1}\right) \% f$, además esta distribución es modelada mediante la Ecuación (2), mostrando a su vez que el comportamiento se preserva bajo diferentes valores de $N$.

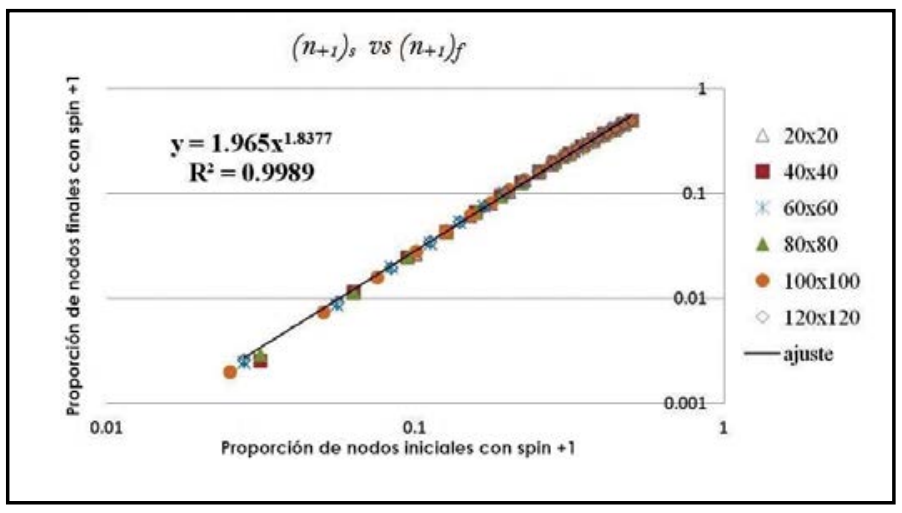

Figura 4. Proporción final de agentes con estado +1 con respecto a su estado inicial en redes de diferente tamaño.
Posteriormente se realizó la variación inicial de nodos $\left(n_{+1}\right)_{\% s}=\left(n_{-1}\right)_{\% s}<=0.5 N$ y al aplicar la dinámica del modelo de simulación para un muestreo de diversos tamaños de redes, se concluye que ambos grupos tienen la misma probabilidad de terminar con mayor número de nodos activos.

\section{Comportamiento de agentes inactivos}

En el segundo análisis son fijados inicialmente los porcentajes de los nodos de la red de la siguiente manera: porcentaje de spin con valor $-1,\left(n_{-1}\right)_{\%}$ igual al $0.5 N \%$, y el porcentaje de spin con valor $+1,\left(n_{+1}\right) \% s$ variable dentro del intervalo $[0,0.5] N$. Con base en y de acuerdo a la evolución del sistema llegando al estado de equilibrio, se muestra que el porcentaje de abstencionistas o spines inactivos $\left(n_{0}\right) \% f$, en relación con el número $\left(n_{+1}\right)_{\% s}$ se distribuye normalmente, aquí resalta que máximo $2.5 \%$ de la población total final queda inactiva, condición que es alcanzada cuando $\left(n_{+1}\right)_{s}=0.3 \mathrm{~N}$, como se puede ver en la Figura 5 . Es de resaltar que el comportamiento mostrado es independiente del valor asignado al tamaño de la red.

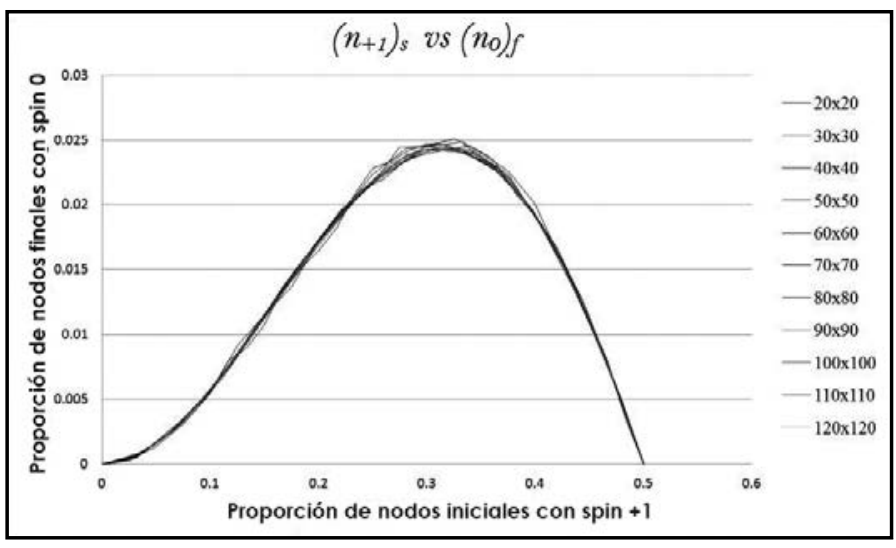

Figura 5. Distribución del número de votos nulos o agentes inactivos $\left(n_{0}\right)_{\% s}$ en relación con el número $\left(n_{+1}\right)_{\% s}$.

Sin embargo, se debe mencionar que el número de nodos inactivos finales cambia dependiendo de la distribución espacial inicial de los nodos con spines -1 y +1 . La Figura 6 muestra la gráfica del porcentaje final de nodos $\left(n_{0}\right)_{\% s}$ en relación con la distribución inicial de spines activos, si estos últimos se distribuyen aleatoriamente en una red de 3600 nodos. La dinámica de distribución inicial de los distintos nodos activos consistió primeramente en seleccionar un valor fijo para $\left(n_{+1}\right)_{\% \text { s }}$ dentro del conjunto $\{0.1 N \%, 0.2 N \%, 0.3 N \%, \ldots, 0.9 N \%\}$, posteriormente se relacionó el valor $\left(n_{+1}\right)_{\%}$ con respecto del valor $\left(n_{-1}\right)_{\% s}$, de tal manera que si $\left(n_{+1}\right)_{\% s}=0.1 N \%$, entonces $\left(n_{-1}\right)_{\% s}=0.9 N \%, 0.8 N \%, 0.7 N \%, \ldots, 0.1 N \%$, si $\quad\left(n_{+1}\right)_{\% s}=0.2 N$, 


\section{InVESEIGACIÓn

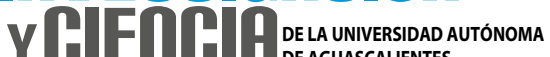 DE AGUASCALIENTES}

entonces $\left(n_{-1}\right)_{\% s}=0.8 N \%, 0.7 N \%, \ldots, 0.1 N \%$, este proceso de asignación concluye cuando se alcanzan los valores $\left(n_{+1}\right)_{\% s}=0.1 \mathrm{~N}$ y $\left(n_{-1}\right)_{\% s}=0.1 \mathrm{~N} \%$.

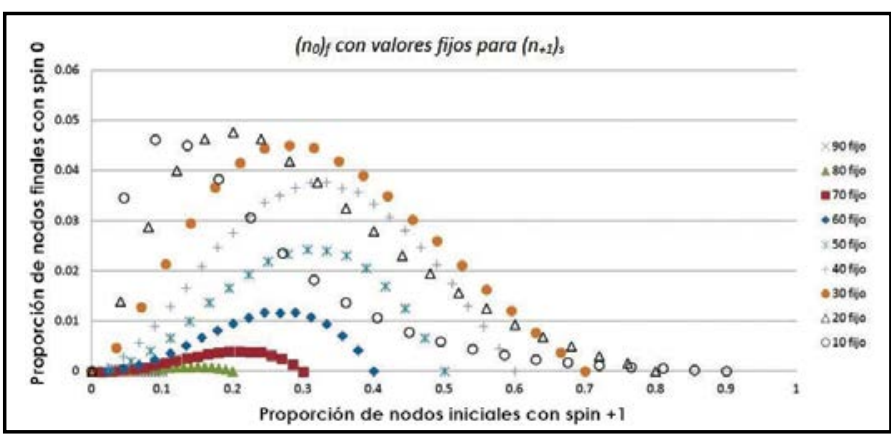

Figura 6. Porcentaje final de agentes con estado 0 con respecto al porcentaje inicial de agentes activos variando sus respectivas proporciones.

De acuerdo con la evolución del sistema bajo las condiciones iniciales comentadas $y$ verificando la Figura 6 , se observa que el valor máximo $\left(n_{0}\right) \%$ de agentes inactivos locales alcanzado es aproximadamente el $5 \%$ del tamaño $N$ de la red, valor que es alcanzado al asignar $\left(n_{+1}\right)_{s}=0.2 N \%$ y $\left(n_{-1}\right)_{s}=0.2 N \%$. Esto da muestra de que la cantidad de agentes inactivos depende de la proporción inicial de nodos con spin con valor -1 y +1 . De la Ecuación 1 y Figura 5 se puede inferir que este comportamiento se hereda para cualquier red de tamaño $N$.

\section{Número de ciclos que definen el estado de equilibrio} Se considera que un sistema se encuentra en su estado de equilibrio cuando se llega al ciclo $n$ tal que para el ciclo $n+1$ ya no es posible que cualquier nodo cambie su estado actual. El ciclo $n$ define entonces el valor de ciclos finales $C_{f}$.

En la Figura 7 se muestra el número $n$ de ciclos $C_{f}$ para conseguir el estado de equilibrio de diversos sistemas con $N$ variable y con una distribución inicial fija de $\left(n_{+1}\right) \%$ s en $0.5 N \%$ y un valor variable para $\left(n_{-1}\right)_{\%}$ dentro del conjunto $\{0.05 \mathrm{~N} \%, 0.1 \mathrm{~N} \%, 0.15 \mathrm{~N} \%$, $0.2 \mathrm{~N} \%, \ldots, 0.5 \mathrm{~N} \%\}$.

Se puede observar de este comportamiento que el número de ciclos para llegar al estado de equilibrio es proporcional al tamaño de la red. Este hecho queda reflejado en la Ecuación 3.

$$
C_{f}=1.9329 * \ln (N)-3.3343
$$

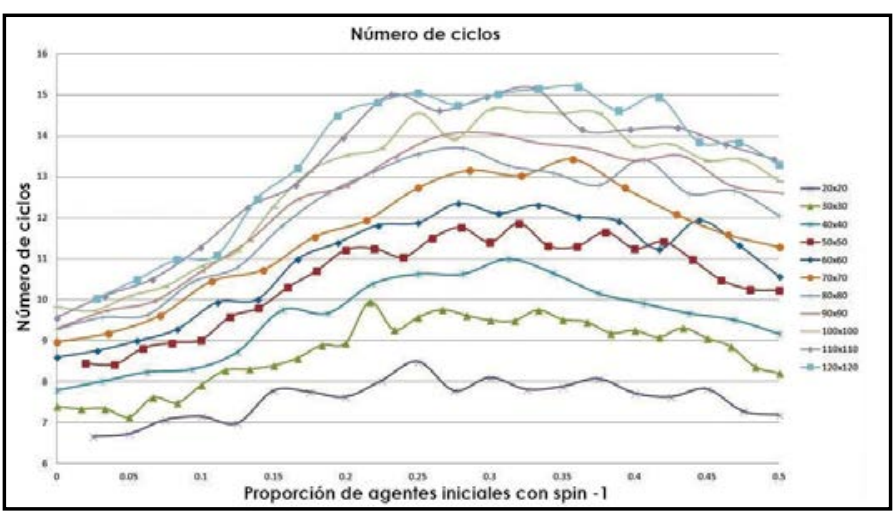

Figura 7. Número de ciclos que determinan el estado de equilibrio en diversas redes.

Modelos de la formación de la opinión, como los expuestos por Chen y Redner (2005) reportan un número grande de ciclos para conseguir su equilibrio, hecho que difiere de lo expuesto en este trabajo, tal como se muestra en la Figura 7.

\section{DISCUSIÓN}

El modelo propuesto en este trabajo es aplicado a sistemas electorales bipartidistas, como un primer acercamiento para simular el posible efecto del voto duro sobre el voto nulo, debido a que un sistema de dos partidos simplifica el estudio de este fenómeno, al reducir el número de variables agregadas al modelo. Por otro lado, podemos afirmar que este estudio podría ser un antecedente para futuras investigaciones en las que se consideren más contendientes en el proceso de simulación.

Otra razón por la cual se enfoca este estudio a sistemas bipartidistas, es porque estos prevalecen en países como Estados Unidos, Australia, Puerto Rico y Belice, por mencionar algunos, y aunque algunos autores exponen sus desventajas y proponen la transformación del sistema a uno multipartidista, estos aportan diversos beneficios a las naciones, los cuales han sido mencionados en este trabajo, como lo expone Lijphart (1994).

En México, en diversas ocasiones las contiendas electorales se reducen a votaciones de dos candidatos, debido a la formación de coaliciones - la falta de participación de ciertos partidos en algunos municipios o estados. En tales casos, el modelo propuesto en este trabajo podría resultar un ejercicio pertinente al simular el posible efecto del voto duro sobre el voto nulo. 


\section{4}

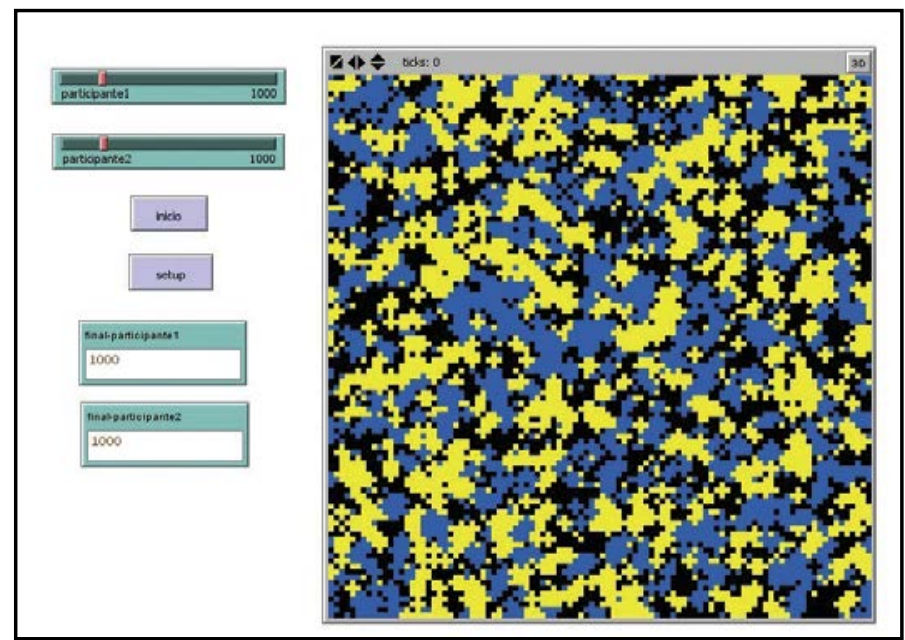

Figura 8. Red electoral general a través de MetLogo. Imagen elaborada por los autores.

Se propone una regla alternativa que evita generar fronteras entre los agentes, comúnmente denominadas franjas; asimismo, evita la existencia de un consenso. Como resultado de la dinámica del modelo ocurre la transformación de la opinión, únicamente del agente observado, a diferencia de la regla mayoritaria propuesta por Galam (1999), en la cual el agente observado y sus cuatro vecinos, todos, cambian al mismo estado, además busca el consenso y su dinámica genera franjas.

En nuestro modelo el número de ciclos necesarios para lograr el equilibrio es menor que en otros modelos de formación de la opinión, como los expuestos por Chen y Redner (2005). Esta regla podría ser una alternativa en el proceso de simulación de la formación de la opinión.
CONCLUSIONES

El modelo presentado muestra un primer acercamiento para explicar el comportamiento del voto nulo intencionado en las contiendas electorales a través del voto duro, basado en un ámbito electoral democrático; hay que recordar que existen diferentes variables reales de las que depende la modelación. Sin embargo, solo se está simulando la interpretación de un modelo que considera la votación efectiva de una contienda. En este caso los participantes con voto activo y preferencia política afectan a la concentración final de votantes con su misma preferencia de forma potencial (Ecuación 2). La afectación del voto activo con preferencia queda explicada por el número de ciclos que definen la estabilidad en un sistema. Un efecto visualizado durante la evolución del sistema y preservado para cualquier tamaño $N$ es el hecho de que algunos agentes de la red no muestran preferencia alguna, esto queda explicado por la consecuencia que genera un agente rodeado de manera equitativa por agentes activos de diferentes preferencias; es así como el votante opta por anular su voto, esto es logrado con mayor frecuencia cuando se tiene una concentración espacial inicial de votantes activos con preferencia de $20 \%$ para ambos simpatizantes.

Por otro lado, el número de ciclos sí es afectado por el tamaño de la red; sin embargo, el orden del número máximo de ciclos que tiene que transcurrir en un sistema para tomar una decisión es de 24 (Figura 7). 


\section{LITERATURA CITADA}

- ADJEl, J. K. Ethnicity and Voting Behavior in the Ashanti and Volta Regions of Ghana: A Cramp in the Wheel of a Fledgling Democracy? Journal of Global Initiatives: Policy, Pedagogy, Perspective, 7(1): 1-14, 2012.

- ALDRICH, J. H. Why Parties? The Origin and Transformation of Political Parties in America. Chicago, US: The University of Chicago Press, 1995.

- AlESINA, A. y SPEAR, S. E. An Overlapping Generation Model of Electoral Competition. Journal of Public Economics, 37(3): 359-379, 1988.

- BALANKIN, A. S. et al. Effect of initial concentration and spatial heterogeneity of active agent distribution on opinion dynamics. Physica A: Statistical Mechanics and its Applications, 390(21): 3876-3887, 2011

- CHEN, P. y REDNER, S. Majority rule dynamics in finite dimensions. Physical Review E, 71 (3): 036101, 2005.

- GALAM, S. Application of statistical physics to politics. Physica A, 274, 132-139, 1999.

- GIDDENS, A. Más allá de la izquierda y la derecha: El futuro de las políticas radicales. Madrid, España: Cátedra, 1996.

- HUANG, G. et al. The strength of the minority. Physica A, 387 , 4665-4672, 2008.

- KNOKE, D. y YANG, S. Social Network Analysis (Quantitative Applications in the Social Sciences). US: Sage Publications, 2008.

- LAMBIOTTE, R. et al. Majority model on a network with communities. Physical Review E, 75, 030101 (R), 2007.

- LIJPHART, A. Electoral Systems and Party Systems: A study of twenty-seven Democracies 1945-1990. Oxford, UK: Oxford University Press, 1994.

- MAINWARING, S. y SCULLY, T. R. Introduction: Party Systems in Latin America. En S. Mainwaring \& T. R. Scully (Eds.), Building Democratic Institutions: Party Systems in Latin America (1-34). Stanford, CA, US: Stanford University Press, 1995.

- MIROIU, A. Characterizing majority rule: from profiles to societies. Economics Letters, 85(3): 359-363, 2004.
- $\quad$ PABJAN, B. y PEKALSKI, A. Model of opinion forming and voting. Physica A, 38, 6183, 2008.

- POPKIN, S. L. The Reasoning Voter: Communication and Persuasion in Presidential Campaigns. Chicago, US: The University of Chicago Press, 1991.

- RAE, D. The Political Consequences of Electoral Laws. New Haven, Connecticut, US: Yale University Press, 1969.

- REGENWETter, M. et al. A general concept of majority rule. Mathematical Social Sciences, 43(3): 405-428, 2002.

- ROSENBLUM, N. L. On the Side of the Angels: An Appreciation of Parties and Partisanship. Princeton, NJ, US: Princeton University Press, 2008.

- SIRVENT, C. y DELGADILLO, F. Realineamiento del voto y nuevo orden electoral 1997. El Nacional, p. 2, jueves 10 de abril de 1997.

- $\quad$ TIDEMAN, T. N. A Majority-Rule Characterization with Multiple Extensions. Social Choice and Welfare, 3(1): 17-30, 1986.

- TOIVONEN, R. et al. A comparative study of social network models: Network evolution models and nodal attribute models. Social Networks, 31 (2009): 240-254, 2009.

- $\quad$ TRAVIESO, G. y COSTA, L. F. Spread of opinions and proportional voting. Physical Review E, 74, $036112,2006$.

- TSALLIS, C. A majority rule model: real space renormalization group solution and finite size scaling. Journal de Physique Letters France, 43, L471-L476, 1982.

- VILELA, A. L. M. y MOREIRA, F. G. B. Majority-vote model with different agents. Physica A: Statistical Mechanics and its Applications, 388(19): 4171-4178, 2009.

- $\quad$ WASSERMAN, S. y FAUST, K. Social Network Analysis: Methods and Applications. Cambridge, UK-NY, US: Cambridge University Press, 1994.

- WILHEIM, G. Democracy in the digital age: challenges to political life in cyberspace. NY, US-London, UK: Routledge, 2000. 